KL-TH $98 / 3$

\title{
Exactly solvable dynamical systems in the neighborhood of the Calogero model
}

\author{
Oliver Haschke and Werner Rühl \\ Department of Physics, University of Kaiserslautern, P.O.Box 3049 \\ 67653 Kaiserslautern, Germany
}

\begin{abstract}
The Hamiltonian of the $N$-particle Calogero model can be expressed in terms of generators of a Lie algebra for a definite class of representations. Maintaining this Lie algebra, its representations, and the flatness of the Riemannian metric belonging to the second order differential operator, the set of all possible quadratic Lie algebra forms is investigated. For $N=3$ and $N=4$ such forms are constructed explicitly and shown to correspond to exactly solvable Sutherland models. The results can be carried over easily to all $N$.
\end{abstract}

April 1998 


\section{Introduction: The Calogero model}

The Calogero model [1] is a quantum mechanical system of $N$ particles moving on a line. It is completely integrable and possesses both fermionic (totally antisymmetric) and bosonic (totally symmetric) solutions. For a review see [2]. In [3] it has been shown that it can be reduced to a representation theory problem of Lie algebras, so that both eigenvalues and eigenfunctions can be extracted from finite dimensional representations of these algebras.

The idea that exact solvability and Lie algebraic representation might be connected was presented in [4], which dealt with exactly and quasiexactly solvable elementary models. Their Hamiltonians were rewritten as quadratic polynomials in Lie algebra differential operators. In this article it was also discussed first whether this procedure could be inverted: Find new exactly solvable models from Lie algebras of differential operators. Usually this leads to kinetic energy terms which are Laplace-Beltrami operators on a curved space. So the task arises to construct quadratic polynomials from the Lie algebras with the constraint that the curvature tensor vanishes identically on some dynamically accessible domain ("flat quadratic Lie algebraic forms"). In order to maintain the notion of exact integrability, however, the nontrivial but curvature-free Laplace-Beltrami operator should be transformed into a standard Laplace operator by an explicitly known diffeomorphism. This has to be found from nonlinear partial differential equations. In the neighborhood of the Calogero models for $N=3$ and $N=4$ such approach is feasible as we shall show in this article. The solutions we give depend on a deformation parameter $s$ ranging over the full real axis, the case $s=0$ belonging to the Calogero models themselves.

In the last year two further articles have appeared dealing with the algebraization of solvable models [5, 6]. We hope that these results can be used to simplify the inverse issue, the construction of new solvable dynamical models.

Let us now outline the algebraization of the standard $(\mathrm{sl}(\mathrm{N}))$ Calogero model, since we use it in the sequel. The Hamiltonian is

$$
H=+\frac{1}{2}\left[-\Delta+\omega^{2} x^{2}\right]+\sum_{i<j} \frac{g}{\left(x_{i}-x_{j}\right)^{2}}
$$

where $x \in \mathbb{R}_{N}$ and

$$
x^{2}=\sum_{i=1}^{N} x_{i}^{2}
$$

$g$ is a coupling constant

$$
g=\nu(\nu-1) \geq-\frac{1}{4}
$$

so that

$$
\nu=\frac{1}{2} \pm\left(g+\frac{1}{4}\right)^{\frac{1}{2}}
$$


is an integer. If $\nu$ is odd (even) we find totally antisymmetric (symmetric) solutions.

We factorize the solutions as

$$
\psi(x)=V(x)^{\nu} e^{-\frac{1}{2} \omega x^{2}} P(x)
$$

where $V(x)$ is the Vandermonde determinant

$$
V(x)=\prod_{i<j}\left(x_{i}-x_{j}\right)
$$

and $P(x)$ is a polynomial which is totally symmetric in $\left\{x_{i}\right\}_{1}^{N}$. If $\psi$ is a solution of

$$
H \psi=E \psi
$$

then $P$ has to solve

$$
h P=\epsilon P
$$

with

$$
h=-\Delta+2 \omega \sum_{i=1}^{N} x_{i} \frac{\partial}{\partial x_{i}}-\nu \sum_{i \neq j} \frac{1}{x_{i}-x_{j}}\left(\frac{\partial}{\partial x_{i}}-\frac{\partial}{\partial x_{j}}\right)
$$

and

$$
\epsilon=2 E-N \omega-\nu N(N-1) \omega
$$

Next we introduce elementary symmetric functions (following [3])

$$
\prod_{i=1}^{N}\left(1+x_{i} t\right)=\sum_{n=0}^{N} \sigma_{n}(x) t^{n}\left(\sigma_{0}=1\right)
$$

For each element $g \in S_{N}$ (the symmetric group of $N$ elements) we have a "sector"

$$
\begin{gathered}
E_{g}=\left\{x_{i_{1}}<x_{i_{2}}<x_{i_{3}}<\ldots<x_{i_{N}}\right. \\
\left.(1,2,3, \ldots N) \underset{ }{\rightarrow}\left(i_{1}, i_{2}, \ldots i_{N}\right)\right\} \\
\quad \subset \mathbb{R}_{N}
\end{gathered}
$$

so that the map

$$
\sigma: x \rightarrow\left\{\sigma_{n}\right\}_{n=1}^{N}
$$

is a diffeomorphism. This follows from the fact that the Jacobian

$$
\mathcal{M}_{a i}=\frac{\partial \sigma_{a}}{\partial x_{i}}
$$

has

$$
\operatorname{det} \mathcal{M}=V(x)(-1)^{\left[\frac{N}{2}\right]}
$$


where $V(x)$ is the Vandermonde determinant. Its square is a polynomial $v\left(\sigma_{1}, \ldots, \sigma_{N}\right)$. The domain $D_{+} \subset \mathbb{R}_{N}$ where $v>0$ is the image $\sigma\left(E_{g}\right)$ for each $g \in S_{N}$.

Next we eliminate the centre-of-mass motion by the substitution

$$
x_{i} \rightarrow y_{i}=x_{i}-\frac{1}{N} \sigma_{1}
$$

Since

$$
\prod_{i=1}^{N}\left(1+y_{i} t\right)=1+\sum_{n=2}^{N} \tau_{n} t^{n}
$$

implies

$$
\tau_{k}=\sigma_{k}+\sum_{r=0}^{k-1}\left(\begin{array}{c}
N-r \\
k-r
\end{array}\right)\left(-\frac{\sigma_{1}}{N}\right)^{k-r} \sigma_{r}
$$

we have

$$
\operatorname{det} \tilde{\mathcal{M}}=V(x)(-1)^{\left[\frac{N}{2}\right]}
$$

for

$$
\tilde{\mathcal{M}}_{a, i}=\left\{\frac{\partial \sigma_{1}}{\partial x_{i}}, \frac{\partial \tau_{2}}{\partial x_{i}}, \ldots \frac{\partial \tau_{N}}{\partial x_{i}}\right\}
$$

Therefore we can use

$$
\left\{\sigma_{1}, \tau_{2}, \ldots, \tau_{n}\right\}
$$

as coordinates on each $E_{g}$.

On $E_{g}$ we have the Laplacian (see [3])

$$
\Delta=N \frac{\partial^{2}}{\partial \sigma_{1}^{2}}+\sum_{j, k=2}^{N} A_{j k} \frac{\partial^{2}}{\partial \tau_{j} \partial \tau_{k}}+\sum_{i=2}^{N} B_{i} \frac{\partial}{\partial \tau_{i}}
$$

with

$$
\begin{gathered}
A_{j k}=\frac{1}{N}(N-j+1)(k-1) \tau_{j-1} \tau_{k-1} \\
+\sum_{l \geq \max (1, k-j)}(k-j-2 l) \tau_{j+l-1} \tau_{k-l-1} \\
B_{i}=-\frac{1}{N}(N-i+2)(N-i+1) \tau_{i-2}
\end{gathered}
$$

where we insert

$$
\tau_{0}=1, \tau_{i}=0 \text { for all } i \notin\{0,2,3, \ldots, N\}
$$


For $h(1.8)$ we have then

$$
\begin{array}{r}
h=-N \frac{\partial^{2}}{\partial \sigma_{1}^{2}}+2 \omega \sigma_{1} \frac{\partial}{\partial \sigma_{1}}-\sum_{j, k=2}^{N} A_{j k} \frac{\partial^{2}}{\partial \tau_{j} \partial \tau_{k}} \\
+2 \omega \sum_{j=2}^{N} j \tau_{j} \frac{\partial}{\partial \tau_{j}}+\left(\frac{1}{N}+\nu\right) \sum_{i=2}^{N}(N-i+2)(N-i+1) \tau_{i-2} \frac{\partial}{\partial \tau_{i}}
\end{array}
$$

Next we introduce the Lie algebra of operators

$$
J_{o i}=\frac{\partial}{\partial \tau_{i}}, J_{i j}=\tau_{i} \frac{\partial}{\partial \tau_{j}}, \quad i, j \in\{2,3, \ldots, N\}
$$

This algebra has the structure

$$
g l(N-1) \mathrm{s} t_{N-1} \quad\left(t_{N-1} \text { abelian }\right)
$$

We represent it in the polynomial spaces $V_{n}$

$$
\begin{gathered}
V_{n}=\oplus_{m=0}^{n} U_{m} \\
U_{m}=\operatorname{span}\left\{\tau_{2}^{n_{2}}, \tau_{3}^{n_{3}}, \ldots, \tau_{N}^{n_{N}}, \sum_{i=2}^{N} n_{i}=m\right\} \\
\operatorname{dim} V_{n}=\left(\begin{array}{c}
n+N-1 \\
n
\end{array}\right)
\end{gathered}
$$

so that

$$
V_{0} \subset V_{1} \subset V_{2} \subset \ldots
$$

forms a flag of polynomial spaces. The algebra (1.25) can be obtained from a maximally degenerate representation of $\mathrm{sl}(\mathrm{N})$ (Young tableau with only one row).

Finally we separate off the centre-of-momentum motion by

$$
h=-N \frac{\partial^{2}}{\partial \sigma_{1}^{2}}+2 \omega \sigma_{1} \frac{\partial}{\partial \sigma_{1}}+h_{\mathrm{rel}}
$$

and obtain the relative motion Hamiltonian $h_{\text {rel }}$ as a quadratic polynomial in the eveloping algebra of (1.25), (1.26) (see [3]).

\section{The inverse issue}

We consider a Lie algebra spanned by a basis $\left\{J_{\alpha}\right\}_{1}^{M}$ being respresented in a flag of polynomial spaces

$$
\begin{array}{r}
V_{0} \subset V_{1} \subset V_{2} \subset \ldots \\
n_{k}=\operatorname{dim} V_{k}<\infty \\
1=n_{0}<n_{1}<n_{2}<\ldots
\end{array}
$$


and such that

$$
J^{\alpha} V_{n} \subset V_{n}
$$

Then the spectrum and the eigenvectors of the form

$$
\sum_{\alpha, \beta=1}^{M} C_{\alpha \beta} J_{\alpha} J_{\beta}+\sum_{\alpha=1}^{M} G_{\alpha} J_{\alpha}
$$

with constant coefficients $\left\{C_{\alpha \beta}, G_{\alpha}\right\}$ can be calculated from representation theory.

The quadratic term in (2.3) defines a Laplace-Beltrami operator which we want to relate with a standard Laplacian from the kinetic energy term in a Schrödinger equation. For this purpose it must have zero curvature. So the first task is to find the flat quadratic Lie algebraic forms

$$
\sum_{\alpha, \beta} C_{\alpha \beta} J_{\alpha} J_{\beta}
$$

This is a highly non-trivial problem.

We assume that a curvature free quadratic form

$$
\sum_{\alpha, \beta} C_{\alpha \beta}^{(o)} J_{\alpha} J_{\beta}
$$

(one point in quadratic Lie algebraic form space) is known. For $t \in \mathbb{R}_{M}$ define

$$
U(t)=\exp \left\{\sum_{\alpha} t_{\alpha} J_{\alpha}\right\}
$$

and its action

$$
U(t) J_{\alpha} U(t)^{-1}=\sum_{\beta} T_{\alpha \beta}(t) J_{\beta}
$$

Then

$$
\begin{array}{r}
U(t)\left(\sum_{\alpha \beta} C_{\alpha \beta}^{(0)} J_{\alpha} J_{\beta}\right) U(t)^{-1} \\
=\sum_{\alpha \beta} C_{\alpha \beta}(t) J_{\alpha} J_{\beta}
\end{array}
$$

with

$$
C_{\alpha \beta}(t)=\sum_{\gamma, \delta} C_{\gamma \delta}^{(0)} T_{\gamma \alpha}(t) T_{\delta \beta}(t)
$$

defines an $M$-dimensional orbit space of flat quadratic Lie algebraic forms. In the case (1.25), (1.26)

$$
\tau_{k}^{\prime}=U(t) \tau_{k} U(t)^{-1}=\Theta_{k}(t ; \tau)
$$


is an affine linear mapping. The flat quadratic Lie algebraic forms we are searching for are determined up to such Lie algebraic automorphisms.

Assume now that $\left\{C_{\alpha \beta}^{(0)}\right\}$ is contained in a curve $\left\{C_{\alpha \beta}(s)\right\}$ of flat quadratic Lie algebraic forms for $s=0$. Since $\left\{C_{\alpha \beta}^{(0)}\right\}$ is assumed to be flat, there exists a diffeomorphism of some dynamically accessible domain

$$
\tau_{i}=\phi_{i}(\xi), i \in\{1,2,3, \ldots r\}
$$

so that

$$
\sum_{\alpha, \beta} C_{\alpha \beta}^{(0)} J_{\alpha} J_{\beta}=\sum_{i=1}^{r} \frac{\partial^{2}}{\partial \xi_{i}^{2}}+\text { lower order diff. operator }
$$

Then we need a 1-parametric set of diffeomorphisms

$$
\begin{array}{r}
\tau_{i}=f_{i}\left(s ; \tau^{\prime}\right) \\
i \in\{1,2,3, \ldots r\}
\end{array}
$$

so that

$$
\sum_{\alpha, \beta} C_{\alpha \beta} J_{\alpha}(\tau) J_{\beta}(\tau)=\sum_{\alpha, \beta} C_{\alpha \beta}^{(0)} J_{\alpha}\left(\tau^{\prime}\right) J_{\beta}\left(\tau^{\prime}\right)+\text { lower order diff. operator }
$$

Both types of diffeomorphisms (2.11), (2.13) ought to be known explicitly, in order to maintain the concept of "exact solvability".

In the following sections we shall give a curve of forms for the Calogero point $\left\{C_{\alpha \beta}^{(0)}\right\}$ for $N=3$ and $N=4$ satisfying these requirements.

\section{The $N=3$ Calogero model and the flatness constraint}

In the case $N=3$ we obtain the following Lie algebraic operators from (1.25) in lexicographic order

$$
\begin{array}{r}
J_{1}=\frac{\partial}{\partial \tau_{2}}, J_{2}=\frac{\partial}{\partial \tau_{3}}, J_{3}=\tau_{2} \frac{\partial}{\partial \tau_{2}} \\
J_{4}=\tau_{2} \frac{\partial}{\partial \tau_{3}}, J_{5}=\tau_{3} \frac{\partial}{\partial \tau_{2}}, J_{6}=\tau_{3} \frac{\partial}{\partial \tau_{3}}
\end{array}
$$

and from (1.20), (1.21) (then (2.4) belongs to $-h(1.8)$ and $+\Delta(1.20)$ )

$$
\begin{gathered}
C_{16}^{(0)}+C_{25}^{(0)}=-3 \\
C_{44}^{(0)}=+\frac{2}{3} \\
C_{13}^{(0)}=-1
\end{gathered}
$$


all other coefficients are vanishing

$$
\begin{gathered}
C_{14}^{(0)}+C_{23}^{(0)}=C_{36}^{(0)}+C_{45}^{(0)}=0 \\
C_{11}^{(0)}=C_{12}^{(0)}=C_{15}^{(0)}=C_{22}^{(0)}=C_{24}^{(0)}=C_{26}^{(0)}= \\
C_{33}^{(0)}=C_{34}^{(0)}=C_{35}^{(0)}=C_{46}^{(0)}=C_{55}^{(0)}=C_{56}^{(0)}=C_{66}^{(0)}=0
\end{gathered}
$$

In order to find the set $\left\{C_{\alpha \beta}(s)\right\}$ corresponding to a flat quadratic Lie algebraic form we can calculate the curvature tensor, each component of which is a rational function of the $\left\{C_{\alpha \beta}(s)\right\}$. Equating a numerator to zero means that in turn all coefficients of the monomials $\tau_{2}^{n} \tau_{3}^{m}$ vanish. This leads to an enormous number of equations of high polynomial degree in the 21 variables $\left\{C_{\alpha \beta}(s)\right\}$. However, after we discovered a solution this way, we developed a simpler algorithm whose variable set is considerably reduced.

First we take account of the irrelevant automorphisms (2.10) and postulate that (2.13) takes the form

$$
\tau_{i}=\tau_{i}^{\prime}+O(s) \quad i \in\{2, \ldots, N\}
$$

in general. Second we make an ansatz

$$
\begin{array}{r}
\sum_{\alpha, \beta} C_{\alpha \beta}(s) J_{\alpha} J_{\beta}=\sum_{a, b=2}^{N} g_{a b}^{-1}(s ; \tau) \frac{\partial}{\partial \tau_{a}} \frac{\partial}{\partial \tau_{b}} \\
\quad \text { lower order differential operator }
\end{array}
$$

where each $g_{a b}^{-1}(s ; \tau)$ is at most of degree two in $\left\{\tau_{n}\right\}$.

$$
\begin{gathered}
g_{a b}^{-1}(s ; \tau)=\sum_{i, j \in\{0,2,3, \ldots, N\}} \gamma_{a b, i j}(s) \tau_{i} \tau_{j} \\
\left(\tau_{0}=1\right)
\end{gathered}
$$

For $s=0$ we postulate

$$
g_{a b}^{-1}(0 ; \tau)=\left(g^{(0)}\right)_{a b}^{-1}(\tau)
$$

and in particular for $N=3$ from (3.2)-(3.6)

$$
\left(g^{(0)}\right)_{a b}^{-1}=\left(\begin{array}{cc}
-2 \tau_{2} & -3 \tau_{3} \\
-3 \tau_{3} & \frac{2}{3} \tau_{2}^{2}
\end{array}\right)
$$

Third we introduce the concepts of a "dimension" $\lambda_{n}$ for each $\tau_{n}$

$$
\left[\tau_{n}\right]=\lambda_{n}
$$


and of a "signature"

$$
\operatorname{sign}\left(\tau_{2 n}\right)=+1, \quad \operatorname{sign}\left(\tau_{2 n+1}\right)=-1
$$

Then the whole expression (3.8) is (from (3.11)) required to be of dimension $\lambda_{2}^{-1}$ and of sign +1 . The existence of independent dimensions (3.12) for each $\tau_{n}$ is connected with the fact that (2.6), (2.10) permits separate dilation automorphisms

$$
\begin{gathered}
U_{n}(t)=\exp \left\{t \tau_{n} \frac{\partial}{\partial \tau_{n}}\right\} \\
\tau_{n}^{\prime}=e^{t} \tau_{n}, \tau_{m}^{\prime}=\tau_{m}(m \neq n)
\end{gathered}
$$

The parameter $s$ has naturally a dimension (see below)

$$
s:[s]=\lambda_{2}^{-1}
$$

But besides $s$ we need other dimensional quantities for $n \in\{3, \ldots, N\}$

$$
w_{n}:\left[w_{n}\right]=\frac{\lambda_{2}^{\frac{n}{2}}}{\lambda_{n}}
$$

The set

$$
\left\{s, w_{n}\right\}_{n=3}^{N}
$$

contains the "scaling parameters". At the end it will turn out that the diffeomorphism (2.13) can solely be expressed by functions of scale-invariant variables

$$
\begin{aligned}
\xi & =s \tau_{2} \\
\eta_{n} & =w_{n} s^{\frac{n}{2}} \tau_{n}, \quad n \in\{3, \ldots, N\}
\end{aligned}
$$

Using these concepts the most general ansatz for (3.9) is for $N=3$

$$
g_{a b}^{-1}(s ; \tau)=-\left(\begin{array}{l}
2 \tau_{2}+s \tau_{2}^{2}+a_{2} w_{3}^{2} s^{2} \tau_{3}^{2}, 3 \tau_{3}+a_{1} s \tau_{2} \tau_{3} \\
3 \tau_{3}+a_{1} s \tau_{2} \tau_{3},-\frac{2}{3} w_{3}^{-2} \tau_{2}^{2}+a_{3} s \tau_{3}^{2}
\end{array}\right)
$$

Obviously neither $s$ nor $w_{3}$ can be determined from the flatness constraints. In order to determine the three numerical parameters $a_{1}, a_{2}, a_{3}$ we may set

$$
s=1, w_{3}=1
$$

respectively we must obtain (3.11) for

$$
s=0, w_{3}=1
$$

Then it results from flatness

$$
a_{1}=\frac{4}{3}, a_{2}=-\frac{1}{6}, a_{3}=1
$$




\section{The basic diffeomorphism}

The basic diffeomorphism (2.13) is obtained from the system of nonlinear differential equations

$$
g^{-1}(s ; \tau)=\Omega^{T}\left(g^{(0)}\right)^{-1}\left(\tau^{\prime}\right) \Omega
$$

where by derivation of (2.13)

$$
\Omega_{a b}\left(\tau^{\prime}\right)=\frac{\partial \tau_{b}}{\partial \tau_{a}^{\prime}}\left(\tau^{\prime}\right)
$$

We use the scale invariant variables (3.18) with

$$
\eta_{3}=\eta
$$

Then the Laplace-Beltrami operators appearing in (2.14) are

$$
\left(\frac{\partial}{\partial \xi}, \frac{\partial}{\partial \eta}\right) \Gamma(\xi, \eta)\left(\begin{array}{c}
\frac{\partial}{\partial \xi} \\
\frac{\partial}{\partial \eta}
\end{array}\right) \quad \text { (no differentiation of } \Gamma \text { ) }
$$

with

$$
\begin{gathered}
\Gamma(\xi, \eta)=\left(\begin{array}{cc}
-2 \xi-\xi^{2}+\frac{1}{6} \eta^{2}, & -3 \eta-\frac{4}{3} \xi \eta \\
-3 \eta-\frac{4}{3} \xi \eta, & +\frac{2}{3} \xi^{2}-\eta^{2}
\end{array}\right) \\
\Gamma^{(0)}(\xi, \eta)=\left(\begin{array}{cc}
-2 \xi, & -3 \eta \\
-3 \eta, & +\frac{2}{3} \xi^{2}
\end{array}\right)
\end{gathered}
$$

The diffeomorphism (2.13) involves two functions

$$
\begin{aligned}
& \xi=F\left(\xi^{\prime}, \eta^{\prime}\right) \\
& \eta=G\left(\xi^{\prime}, \eta^{\prime}\right)
\end{aligned}
$$

that must be determined from

$$
\Gamma(\xi, \eta)=H^{T} \Gamma^{(0)}\left(\xi^{\prime}, \eta^{\prime}\right) H
$$

where

$$
H=\left(\begin{array}{ll}
\frac{\partial F}{\partial \xi^{\prime}} & \frac{\partial G}{\partial \xi^{\prime}} \\
\frac{\partial F}{\partial \eta^{\prime}} & \frac{\partial G}{\partial \eta^{\prime}}
\end{array}\right)
$$

Imposing the boundary conditions (3.7) the solution of (4.8) is unique and can be given in series form

$$
F(\xi, \eta)=\sum_{\substack{n=0 \\(n+m \geq 1)}}^{\infty} \sum_{m=0}^{\infty}(-1)^{m} \frac{2^{n+3 m-1}(n+2 m-1) !}{n !(2 m) !(2 n+6 m-1) !} \xi^{n} \eta^{2 m}
$$




$$
G(\xi, \eta)=\sum_{n=0}^{\infty} \sum_{m=0}^{\infty}(-1)^{m} \frac{2^{n+3 m+1}(n+2 m) !}{n !(2 m+1) !(2 n+6 m+2) !} \xi^{n} \eta^{2 m+1}
$$

Both functions are entire analytic. The Jacobian $H(4.9)$ is, however, not throughout invertible on the whole $\xi, \eta$-plane.

A summed up form for $F$ and $G$ is obtained after insertion of the functions (see $(1.16),(3.18)$ )

$$
\begin{aligned}
\xi & =s\left(y_{1} y_{2}+y_{2} y_{3}+y_{3} y_{1}\right) \\
\eta & =s^{\frac{3}{2}} y_{1} y_{2} y_{3}
\end{aligned}
$$

namely

$$
\begin{aligned}
F & =2 \sum_{\text {cycl. perm. of } 1,2,3} \sin \left\{\left(\frac{s}{2}\right)^{\frac{1}{2}} y_{i}\right\} \sin \left\{\left(\frac{s}{2}\right)^{\frac{1}{2}} y_{j}\right\} \cos \left\{\left(\frac{s}{2}\right)^{\frac{1}{2}} y_{k}\right\} \\
G & =8^{\frac{1}{2}} \sin \left\{\left(\frac{s}{2}\right)^{\frac{1}{2}} y_{1}\right\} \sin \left\{\left(\frac{s}{2}\right)^{\frac{1}{2}} y_{2}\right\} \sin \left\{\left(\frac{s}{2}\right)^{\frac{1}{2}} y_{3}\right\}
\end{aligned}
$$

From (4.10), (4.11) one can easily get the series expansion for $\operatorname{det} H$

$$
\begin{gathered}
\operatorname{det} H(\xi, \eta)=\sum_{n, m=0}^{\infty} h_{n m} \xi^{n} \eta^{2 m} \\
\left(h_{00}=1\right)
\end{gathered}
$$

but a closed form for $h_{n m}$ has not been obtained. Nevertheless we were able to sum this series as follows.

The square of the Vandermonde determinant gives (at $w_{3}=1$ )

$$
D=4 \xi^{3}+27 \eta^{2}=-s^{3} V(y)^{2}
$$

so that $s>0(s<0)$ implies $D<0(D>0)$ respectively. The separatrix

$$
D=0
$$

separates a domain

$D<0$ : here det $H=0$ can be fulfilled on an infinite number of zero-trajectories $\mathcal{C}_{n}, n \in\{1,2,3 \ldots\}$

from the domain

$D>0$ : where $\operatorname{det} H>0$.

The curves $\mathcal{C}_{n}$ are given by

$$
\mathcal{C}_{n}:\left(1+\frac{3}{2} \frac{\xi}{n^{2} \pi^{2}}\right)^{2}+\frac{D}{8 n^{6} \pi^{6}}=0
$$




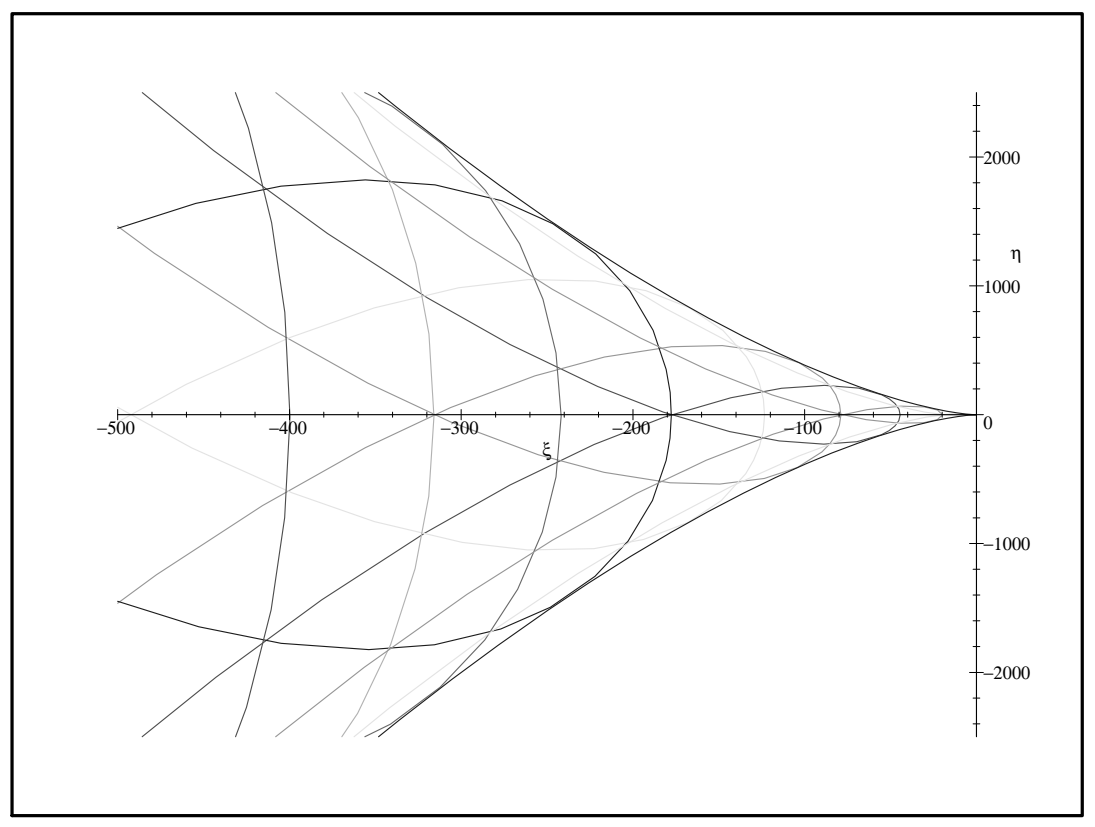

Figure 1: The zerotrajectories $\mathcal{C}_{n}$ and the separatrix in $(\xi, \eta)$-plane

They were first discovered by numerical computations (Fig. 1).

It is obviously suggesting to attempt a Weierstraß product ansatz

$$
\operatorname{det} H(\xi, \eta)=\prod_{n=1}^{\infty}\left[\left(1+\frac{3}{2} \frac{\xi}{n^{2} \pi^{2}}\right)^{2}+\frac{D}{8 n^{6} \pi^{6}}\right]
$$

That this is in fact correct can be verified in the following fashion.

Consider the polynomial

$$
x^{3}+u x+v=0
$$

with its factorized form

$$
\prod_{i=1}^{3}\left(x-\alpha_{i}\right)=0
$$

so the elementary symmetric polynomials of $\{\alpha\}_{i=1}^{3}$ are

$$
\sigma_{1}(\alpha)=0, \sigma_{2}(\alpha)=u, \sigma_{3}(\alpha)=-v
$$

By symmetric evaluation of the product we have

$$
\begin{aligned}
& \prod_{n=-\infty}^{+\infty}\left(1-\frac{\alpha_{i}}{n \pi}\right)=\prod_{n=1}^{\infty}\left(1-\frac{\alpha_{i}^{2}}{n^{2} \pi^{2}}\right) \\
& \text { (symm. } \\
& n \neq 0 \text { ) }
\end{aligned}
$$




$$
=\frac{\sin \alpha_{i}}{\alpha_{i}}
$$

Correspondingly we get

$$
\begin{aligned}
\operatorname{det} H(\xi, \eta) & =\prod_{\substack{n=-\infty \\
(\text { symm. } \\
n \neq 0)}}^{+\infty}\left[1+\frac{3}{2} \frac{\xi}{n^{2} \pi^{2}}+\sqrt{\frac{-D}{8}} \frac{1}{n^{3} \pi^{3}}\right] \\
& =\prod_{i=1}^{3} \frac{\sin \alpha_{i}}{\alpha_{i}}
\end{aligned}
$$

where

$$
u=\frac{3}{2} \xi, v=\sqrt{\frac{-D}{8}}
$$

We can expand (4.24) into a power series of $\alpha_{1}, \alpha_{2}, \alpha_{3}$. Symmetry makes sure that this series can be reformulated in terms of

$$
\sigma_{1}(\alpha), \sigma_{2}(\alpha), \sigma_{3}(\alpha)
$$

Using then (4.22) with (4.25) we obtain a series expansion as in (4.16). By a numerical comparison we can prove their identity.

Finally we consider the separatrix

$$
D=0 \quad \text { implying (say) } \alpha_{3}=0, \alpha_{2}=-\alpha_{1}
$$

This yields by (4.22), 4.25)

$$
-\sigma_{2}(\alpha)=\alpha_{1}^{2}=-\frac{3}{2} \xi
$$

Then

$$
\left.\operatorname{det} H(\xi, \eta)\right|_{D=0}=\left(\frac{\sin \alpha_{1}}{\alpha_{1}}\right)^{2}
$$

implying zeros of $\operatorname{det} H$ at $D=0$ and

$$
-\frac{3}{2} \xi=(n \pi)^{2}, n \in \mathbb{Z}-\{0\}
$$

At this point the zero trajectory $\mathcal{C}_{n}$ touches the separatrix.

The representation of the function $\operatorname{det} H(\xi, \eta)$ simplifies if we return to the relative coordinate variables $y_{1}, y_{2}, y_{3}$. The cubic equation (4.20) suggests the identification

$$
\alpha_{i}=\frac{1}{\sqrt{2}} s^{\frac{1}{2}}\left(y_{j}-y_{k}\right)
$$




$$
(i, j, k \text { cyclic })
$$

Then we find easily

$$
\begin{aligned}
\sigma_{1}(\alpha) & =0 \\
\sigma_{2}(\alpha) & =\frac{3}{2} s \sigma_{2}(y)=\frac{3}{2} \xi \\
\sigma_{3}(\alpha) & =-s^{\frac{3}{2}} \frac{V(y)}{\sqrt{8}}=\mp \sqrt{\frac{-D}{8}}
\end{aligned}
$$

so that $(4.22), 4.25)$ are satisfied. The Vandermonde determinant $V(y)$ was defined in (1.5). In $y$-space the separatrix consists of three straight lines

$$
y_{i}-y_{j}=0, \text { all pairs }(i, j) i \neq j
$$

cutting the plane into six sectors. They correspond to the six elements of the symmetric group $S_{3}$ (see (1.11)-(1.14). From (4.24), (4.30) we see that the inverse image of a zero trajectory $\mathcal{C}_{n}$ consists of six straight lines as well

$$
\frac{1}{\sqrt{2}} s^{\frac{1}{2}}\left(y_{i}-y_{j}\right)=n \pi, n \in \mathbb{N} \text {, all pairs }(i, j) i \neq j
$$

Three of these lines intersect the same sector and these lines intersect each other on the boundary of the sector, i.e. on the separatrix. In Fig. 2 we present the resulting image for the identity element of $S_{3}$ as sector, projected on the $\left(y_{2}, y_{3}\right)$-plane.

\section{The spectrum}

On the linear space (1.27)

$$
V_{n}=\bigoplus_{m=0}^{n} U_{m}
$$

we consider the quadratic operator

$$
-\left(\frac{\partial}{\partial \xi}, \frac{\partial}{\partial \eta}\right) \Gamma(\xi, \eta)\left(\begin{array}{c}
\frac{\partial}{\partial \xi} \\
\frac{\partial}{\partial \eta}
\end{array}\right)
$$

(differentiation of $\Gamma$ included) with $\Gamma$ the matrix (4.5). This operator decomposes in two parts $\mathcal{A}+\mathcal{B}$ so that for all $m$

$$
\mathcal{A} U_{m} \subset U_{m}, \mathcal{B} U_{m+1} \subset U_{m}
$$

Now we span $U_{m}$ by monomials of degree $m$

$$
\sum_{r=0}^{m} a_{r}^{(m)} \xi^{r} \eta^{m-r}
$$




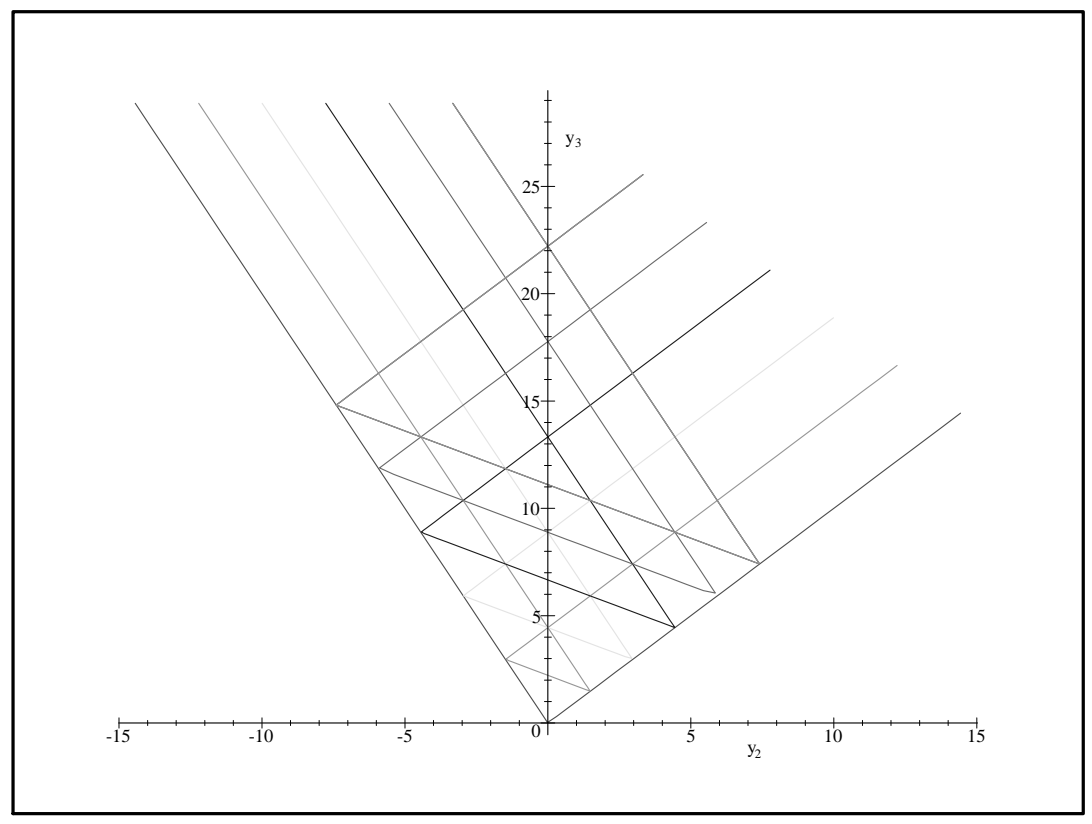

Figure 2: The sector $E_{1}$ in $\left(y_{2}, y_{3}\right)$-plane with zerotrajectories $1 \leq n \leq 5$

then the map $\mathcal{A}$ is

$$
\begin{aligned}
a_{r}^{(m)} \overrightarrow{\mathcal{A}} a_{r}^{(m)}= & {\left[m^{2}+\frac{7}{3} m+\frac{2}{3} r(m-r)\right] a_{r}^{(m)} } \\
& -\frac{2}{3}(m-r+2)(m-r+1) a_{r-2}^{(m)} \\
& -\frac{1}{6}(r+2)(r+1) a_{r+2}^{(m)} \\
a_{r}^{(m)} \underset{\mathcal{B}}{\rightarrow} a_{r}^{\prime(m-1)}= & (r+1)(6 m-4 r-1) a_{r+1}^{(m)}
\end{aligned}
$$

From (5.5), (5.6) we recognize that all spaces $U_{m}$ decompose into direct sums of even and odd parts (the power $r$ of $\xi$ is even or odd, respectively)

$$
\begin{gathered}
U_{m}=U_{m}^{(+)} \oplus U_{m}^{(-)} \\
U_{m}^{(+)}=\operatorname{span}\left\{\xi^{2 r} \eta^{m-2 r}\right\}_{r=0}^{\left[\frac{m}{2}\right]} \\
U_{m}^{(-)}=\operatorname{span}\left\{\xi^{2 r+1} \eta^{m-2 r-1}\right\}_{r=0}^{\left[\frac{m-1}{2}\right]}
\end{gathered}
$$

so that

$$
\begin{aligned}
& \mathcal{A} U_{m}^{( \pm)} \subset U_{m}^{( \pm)} \\
& \mathcal{B} U_{m}^{( \pm)} \subset U_{m-1}^{(\mp)}
\end{aligned}
$$


Correspondingly the set of eigenvalues $\Lambda^{(m)}$ of $\mathcal{A}$ on $U_{m}$ decomposes

$$
\Lambda_{m}=\Lambda_{m}^{(+)} \cup \Lambda_{m}^{(-)}
$$

The eigenvalues on each subspace $U_{m}^{( \pm)}$are non-degenerate for fixed $m$.

For the even part we find

$$
\begin{array}{r}
\Lambda_{m}^{(+)}=\left\{\frac{1}{3}\left[4 m^{2}-(4 r-10) m+4(r-1)^{2}\right]\right. \\
\left.r \in\left\{1,2, \ldots,\left[\frac{m}{2}\right]+1\right\}\right\}
\end{array}
$$

For increasing $r$ the eigenvalues $\lambda_{r}^{(m)}$ decrease. Moreover we find

$$
\begin{aligned}
& \Lambda_{m}^{(-)}=\Lambda_{m}^{(+)}, \quad m \text { odd } \\
& \Lambda_{m}^{(-)}=\Lambda_{m}^{(+)}-\left\{m^{2}+2 m\right\}, \quad m \text { even }
\end{aligned}
$$

where $m^{2}+2 m$ is the smallest eigenvalue of $\Lambda_{m}^{(+)}$.

On $V_{n}$ (5.1) $\mathcal{A}+\mathcal{B}$ has the matrix form

$$
\left(\begin{array}{ccccccc}
\mathcal{A}_{n}, & 0 & & & & & \\
\mathcal{B}_{n}, & \mathcal{A}_{(n-1)} & 0 & & & & \\
0 & \mathcal{B}_{(n-1)} & \mathcal{A}_{(n-2)} & 0 & & & \\
& 0 & \mathcal{B}_{(n-2)} & \mathcal{A}_{(n-3)} & & & \\
& & & & 0 & & \\
& & & & \mathcal{B}_{2} & \mathcal{A}_{1} & 0 \\
& & & & 0 & \mathcal{B}_{1} & \mathcal{A}_{0}
\end{array}\right)
$$

where $\mathcal{A}_{m}\left(\mathcal{B}_{m}\right)$ is the restriction of $\mathcal{A}(\mathcal{B})$ to $U_{m}$. Assume we want to calculate an eigenvector in $V_{n}$ which is not in $V_{m}(m<n)$. If

$$
\Lambda_{m} \cap \Lambda_{m^{\prime}}=\phi \text { for } m \neq m^{\prime}
$$

then

$$
\mathcal{A}_{n} e_{n}=\lambda_{r}^{(n)} e_{n}, \quad e_{n} \in U_{n}
$$

implies for the component $e_{m}$ of the same eigenvector in $U_{m}, m<n$ that

$$
e_{m}=-\left(\mathcal{A}_{m}-\lambda_{r}^{(m)} E\right)^{-1} \mathcal{B}_{(m+1)} e_{(m+1)}
$$

However, (5.15) is not correct. Instead we found an infinite sequence of counter examples. The smallest ones are

$$
\begin{array}{llll}
\lambda_{1}^{(14)}=\lambda_{8}^{(16)}=\frac{868}{3} & \lambda_{1}^{(27)}=\lambda_{15}^{(31)}=1026 \\
\lambda_{2}^{(18)}=\lambda_{9}^{(20)}=\frac{1336}{3} & \lambda_{6}^{(30)}=\lambda_{15}^{(32)}=\frac{3280}{3} \\
\lambda_{3}^{(22)}=\lambda_{10}^{(24)}=636 & \lambda_{5}^{(30)}=\lambda_{12}^{(32)}=\frac{3364}{3} \\
\lambda_{1}^{(22)}=\lambda_{6}^{(24)}=\frac{2068}{3} & \lambda_{2}^{(30)}=\lambda_{7}^{(32)}=\frac{3664}{3} \\
\lambda_{4}^{(26)}=\lambda_{11}^{(28)}=\frac{2584}{3} & \lambda_{2}^{(31)}=\lambda_{16}^{(35)}=\frac{3910}{3}
\end{array}
$$


Two eigenvalues can only coincide if $m_{1}-m_{2}$ is even and $m_{1}, m_{2}$ are large. Consequently for such coinciding pair with $m_{1}<m_{2}, r_{1}<r_{2}$ the inverse

$$
\left(\mathcal{A}_{m_{1}}-\lambda_{r_{2}}^{\left(m_{2}\right)} E\right)^{-1}
$$

does not exist. But in addition

$$
\mathcal{B}_{m_{1}+1} e_{m_{1}+1} \neq 0
$$

since $\mathcal{B}_{m}$ (5.6) is diagonal and its elements are all non-zero.

Thus the eigenvectors in $V_{m_{2}}$ belonging to $\lambda_{r_{2}}^{\left(m_{2}\right)}$ are missing. Any smooth function in $\xi$ and $\eta$ which can be approximated uniformly over compact domains by polynomials due to the Weierstraß approximation theorem cannot be approximated by the eigenpolynomials of the operator (5.2).

This incompleteness can be cured by adding a linear term in the Lie algebra to (5.2), e.g.

$$
+\gamma\left(\xi \frac{\partial}{\partial \xi}+\eta \frac{\partial}{\partial \eta}\right), \gamma \notin \mathbb{Q}
$$

In fact we shall see in the subsequent section that such term is essential for self-adjointness of the Schrödinger operator.

\section{The Schrödinger operator}

We start from (3.19) and consider the operator

$$
Q=-\sum_{a, b} \frac{\partial}{\partial \tau_{a}} g_{a b}^{-1} \frac{\partial}{\partial \tau_{b}}+\gamma \sum_{a} r_{a} \frac{\partial}{\partial \tau_{a}}
$$

where $\gamma \in \mathbb{R}$ is arbitrary and the functions $r_{a}(\tau)$ are linear in $\left\{\tau_{a}\right\}$ and left otherwise open for a while. The spectrum of $Q$ can be calculated with the method of Section 5. Then we may have eigenfunctions $\varphi$ and eigenvalues $\epsilon$

$$
Q \varphi=\epsilon \varphi
$$

By an appropriate choice of a gauge function $\chi$

$$
\varphi=e^{\chi} \psi
$$

we want to transform $Q$ into a standard Schrödinger operator in $\tau$-space

$$
-\Delta \psi+W \psi=\epsilon \psi
$$

Here $\Delta$ is the Laplace-Beltrami operator

$$
\Delta=\frac{1}{\sqrt{g}} \sum_{a, b} \frac{\partial}{\partial \tau_{a}} \sqrt{g} g_{a b}^{-1} \frac{\partial}{\partial \tau_{b}}
$$


and $W$ is a potential depending on $\chi$ and $\gamma$. The determinant $g$ is computed from

$$
g=\left(\operatorname{det}\left\{g_{a b}^{-1}\right\}\right)^{-1}
$$

and comes out from (3.19) as

$$
\begin{array}{r}
g(s ; \tau)^{-1}=-\frac{1}{3}\left\{\left(4 \tau_{2}^{3}+27 \tau_{3}^{2}\right)+2 s \tau_{2}\left(\tau_{2}^{3}+9 \tau_{3}^{2}\right)\right. \\
\left.+2 s^{2} \tau_{2}^{2} \tau_{3}^{2}+\frac{1}{2} s^{3} \tau_{3}^{4}\right\}
\end{array}
$$

This function can be expressed by $\left\{\tau_{a}^{\prime}\right\}$ easily as follows from (4.1)

$$
g\left(s ; \tau\left(\tau^{\prime}\right)\right)^{-1}=-\frac{1}{3}\left(4 \tau_{2}^{\prime 3}+27 \tau_{3}^{\prime 2}\right) \cdot\left(\operatorname{det} \Omega\left(\tau^{\prime}\right)\right)^{2}
$$

After multiplication with appropriate powers of $s$ we obtain from (4.8)

$$
\Gamma(\xi, \eta)=-\frac{1}{3} D\left(\xi^{\prime}, \eta^{\prime}\right)\left(\operatorname{det} H\left(\xi^{\prime}, \eta^{\prime}\right)\right)^{2}
$$

where $\Gamma$ is now the determinant of the matrix (4.5).

In order to fix $\chi$ we start from

$$
\begin{aligned}
e^{-\chi} Q e^{\chi}= & -\sum_{a, b}\left(\frac{\partial}{\partial \tau_{a}}+\frac{\partial \chi}{\partial \tau_{a}}\right) g_{a b}^{-1}\left(\frac{\partial}{\partial \tau_{b}}+\frac{\partial \chi}{\partial \tau_{b}}\right) \\
& +\gamma \sum_{a} r_{a}\left(\frac{\partial}{\partial \tau_{a}}+\frac{\partial \chi}{\partial \tau_{a}}\right)
\end{aligned}
$$

We match the first order differential operator parts in (6.4), (6.5) and $(6.10)$

$$
-2 \sum_{a} \frac{\partial \chi}{\partial \tau_{a}} g_{a b}^{-1}+\gamma r_{b}=-\sum_{a} \frac{\partial}{\partial \tau_{a}}(\ln \sqrt{g}) g_{a b}^{-1}
$$

The integrability condition for this differential equation is

$$
\frac{\partial}{\partial \tau_{a}} \sum_{c} r_{c} g_{c b}=\frac{\partial}{\partial \tau_{b}} \sum_{c} r_{c} g_{c a}
$$

If (6.12) is fulfilled we obtain a function $\rho$ so that

$$
r_{a}=\sum_{b} g_{a b}^{-1} \frac{\partial}{\partial \tau_{b}} \rho
$$

In fact, we can show easily that

$$
\rho=\ln \sqrt{g}
$$


and

$$
\begin{aligned}
& r_{2}=3+2 s \tau_{2} \\
& r_{3}=2 s \tau_{3}
\end{aligned}
$$

fulfill $(6.12)$ and $(6.13)$. Then (6.11) is solved by

$$
\chi=\frac{1}{2}(1+\gamma) \ln \sqrt{g}
$$

and the potential comes out as

$$
\begin{aligned}
W(\tau)= & -\sum_{a, b}\left\{\frac{\partial}{\partial \tau_{a}}\left(g_{a b}^{-1} \frac{\partial \chi}{\partial \tau_{b}}\right)+g_{a b}^{-1} \frac{\partial \chi}{\partial \tau_{a}} \frac{\partial \chi}{\partial \tau_{b}}\right\} \\
& +\gamma \sum_{a} r_{a} \frac{\partial \chi}{\partial \tau_{a}}
\end{aligned}
$$

Due to 6.13$), 6.15)$ the first term in the potential is a constant and we get

$$
W(\tau)=\frac{1}{4}\left(\gamma^{2}-1\right) \sum_{a, b} g_{a b}^{-1} \frac{\partial \ln \sqrt{g}}{\partial \tau_{a}} \frac{\partial \ln \sqrt{g}}{\partial \tau_{b}}+\text { const }
$$

The wave functions

$$
\psi=e^{-\chi} \varphi=g^{-\frac{1}{4}(1+\gamma)} \varphi
$$

have a zero of order $\frac{1}{2}(1+\gamma)$ on each trajectory $\mathcal{C}_{n}$ and on the separatrix which can be seen from

$$
g^{-\frac{1}{2}}=\text { const. }\left|\prod_{i<j} \sin \sqrt{\frac{s}{2}}\left(y_{i}-y_{j}\right)\right|
$$

which follows from (4.17), (4.24), 4.30) and (6.9). Moreover the spectral degeneracy is lifted if $\gamma$ is irrational (and $s=1$, say) due to the form (6.15) of $r_{2}$ and $r_{3}$ (compare with (5.20)). Thus we conclude that

(1) for $\gamma=0$ the Schrödinger operator is only formally selfadjoint, since its eigenfunctions form an incomplete set;

(2) for $\gamma>0$ the Schrödinger operator is self-adjoint if $\gamma \notin \mathbb{Q}$ at least. For (see $(1.3))$

$$
\gamma=2 \nu-1, \quad \nu \in \mathbb{Z}
$$

insertion of (6.20) into (6.18) gives the Sutherland model [7], which is known to be exactly soluble.

The wavefunctions $\psi$ have support on any minimal triangle of a sector $E_{g}$ in $y$-space. This triangle is bounded by three trajectories

$$
\begin{array}{r}
\mathcal{C}_{n_{1}}, \mathcal{C}_{n_{2}}, \mathcal{C}_{n_{3}}: \\
n_{1} \leq n_{2} \leq n_{3}, n_{3}=n_{1}+n_{2} \pm 1
\end{array}
$$




\section{$7 \quad$ The $N=4$ Calogero model}

The Calogero model for $N=3$ is still too special to draw general conclusions from it, e.g. its Hamiltonian (11.1) is separable. In fact we were able to find flat quadratic Lie algebraic forms also for $N=4$. With $s, w_{3}, w_{4}$ as in (3.16), (3.17) we make the ansatz

$$
\begin{aligned}
& g_{22}^{-1}=-2 \tau_{2}-\left(\tau_{2}^{2}+a_{1} w_{4} \tau_{4}\right) s \\
&-\left(a_{2} w_{3}^{2} \tau_{3}^{2}-a_{3} w_{4} \tau_{2} \tau_{4}\right) s^{2} \\
&-a_{4} w_{4}^{2} \tau_{4}^{2} s^{3} \\
& g_{23}^{-1}=- 3 \tau_{3}-a_{5} \tau_{2} \tau_{3} s-a_{6} w_{4} \tau_{3} \tau_{4} s^{2} \\
& g_{24}^{-1}=- 4 \tau_{4}-\left(a_{7} \tau_{2} \tau_{4}+a_{8} w_{4}^{-1} w_{3}^{2} \tau_{3}^{2}\right) s \\
&-a_{9} w_{4} \tau_{4}^{2} s^{2} \\
& g_{33}^{-1}=-4 w_{4} w_{3}^{-2} \tau_{4}+w_{3}^{-2} \tau_{2}^{2} \\
&-\left(a_{10} w_{4} w_{3}^{-2} \tau_{2} \tau_{4}+a_{11} \tau_{3}^{2}\right) s \\
&-a_{12} w_{4}^{2} w_{3}^{-2} \tau_{4}^{2} s^{2} \\
& g_{34}^{-1}=+\frac{1}{2} w_{4}^{-1} \tau_{2} \tau_{3}-a_{13} \tau_{3} \tau_{4} s \\
& g_{44}^{-1}=-2 w_{4}^{-1} \tau_{2} \tau_{4}+\frac{3}{4} w_{3}^{2} w_{4}^{-2} \tau_{3}^{2}-a_{14} \tau_{4}^{2} s
\end{aligned}
$$

Here $w_{3}$ and $w_{4}$ can be scaled to one by the automorphisms (3.14), (3.15). Setting then $s=0$ the Calogero Riemannian for $N=4$ appears.

We have to solve the equations resulting from equating all six components of the curvature tensor to zero. These are polynomial equations and depend on the fourteen variables $\left\{a_{\alpha}\right\}$ of (7.1) $-(7.6)$. The number of equations lies somewhere between $10^{3}$ and $10^{4}$. Using the seventy-eight variables $\left\{C_{\alpha \beta}\right\}$ from (2.4) the number of equations would have been considerably bigger still. We solved the equations by solving first all equations with less than thirty terms and inserting then the result into the bulk of the equations. This trivialized almost all of them. The result is

$$
\begin{array}{ll}
a_{1}=3 a_{7}-5 & a_{8}=-\frac{3}{8} a_{7}+\frac{5}{8} \\
a_{2}=-\frac{3}{16} a_{7}^{2}+\frac{5}{8} a_{7}-\frac{11}{16} & a_{9}=-\frac{1}{2} a_{7}^{2}+\frac{3}{2} a_{7}-1 \\
a_{3}=\frac{1}{2} a_{7}^{2}-a_{7}+\frac{1}{2} & a_{10}=a_{7}-1 \\
a_{4}=-\frac{1}{4} a_{7}^{3}+a_{7}^{2}-\frac{5}{4} a_{7}+\frac{1}{2} & a_{11}=1 \\
a_{5}=-\frac{1}{4} a_{7}+\frac{7}{4} & a_{12}=-\frac{1}{4} a_{7}^{2}+\frac{1}{2} a_{7}-\frac{1}{4} \\
a_{6}=\frac{1}{8} a_{7}^{2}-\frac{1}{2} a_{7}+\frac{3}{8} & a_{13}=\frac{1}{4} a_{7}+\frac{3}{4} \\
a_{7}=a_{7} & a_{14}=-a_{7}+3
\end{array}
$$


Given the Riemannian (7.1)-(7.7) and the unperturbed form

$$
\Gamma^{(0)}=\left(\begin{array}{ccc}
-2 \xi^{\prime} & -3 \eta_{3}^{\prime} & -4 \eta_{4}^{\prime} \\
-3 \eta_{3}^{\prime} & -4 \eta_{4}^{\prime}+\xi^{\prime 2} & \frac{1}{2} \xi^{\prime} \eta_{3}^{\prime} \\
-4 \eta_{4}^{\prime} & \frac{1}{2} \xi^{\prime} \eta_{3}^{\prime} & -2 \xi^{\prime} \eta_{4}^{\prime}+\frac{3}{4} \eta_{3}^{\prime 2}
\end{array}\right)
$$

we can solve (4.8) in terms of three functions

$$
\begin{aligned}
\xi & =F\left(\xi^{\prime}, \eta_{3}^{\prime}, \eta_{4}^{\prime}\right) \\
\eta_{3} & =G_{3}\left(\xi^{\prime}, \eta_{3}^{\prime}, \eta_{4}^{\prime}\right) \\
\eta_{4} & =G_{4}\left(\xi^{\prime}, \eta_{3}^{\prime}, \eta_{4}^{\prime}\right)
\end{aligned}
$$

with the Jacobian matrix

$$
H=\left(\begin{array}{lll}
\frac{\partial F}{\partial \xi^{\prime}} & \frac{\partial G_{3}}{\partial \xi^{\prime}} & \frac{\partial G_{4}}{\partial \xi^{\prime}} \\
\frac{\partial F}{\partial \eta_{3}} & \frac{\partial G_{3}}{\partial \eta_{3}} & \frac{\partial G_{4}}{\partial \eta_{3}} \\
\frac{\partial F}{\partial \eta_{4}^{\prime}} & \frac{\partial G_{3}}{\partial \eta_{4}^{\prime}} & \frac{\partial G_{4}}{\partial \eta_{4}^{\prime}}
\end{array}\right)
$$

We set

$$
w_{3}=w_{4}=1
$$

We find that only $F$ depends on $a_{7}$ linearly

$$
F=F_{1}+a_{7} F_{2}
$$

and that

$$
F_{2}=\frac{1}{2} G_{4}
$$

Consequently $\operatorname{det} H$ is independent of $a_{7}$. The functions $F_{1}, G_{3}, G_{4}$ can be expressed in summed up form correspondingly to (4.14), (4.15)

$$
\begin{aligned}
& F_{1}=\frac{1}{2} \sum_{\text {perm. of }\{1, \ldots, 4\}} \\
& \sin \left\{\left(\frac{s}{2}\right)^{\frac{1}{2}} y_{i}\right\} \sin \left\{\left(\frac{s}{2}\right)^{\frac{1}{2}} y_{j}\right\} \\
& \times \cos \left\{\left(\frac{s}{2}\right)^{\frac{1}{2}} y_{k}\right\} \cos \left\{\left(\frac{s}{2}\right)^{\frac{1}{2}} y_{l}\right\} \\
& G_{3}=\frac{2^{\frac{1}{2}}}{3} \sum_{\text {perm. of }\{1, \ldots, 4\}} \quad \sin \left\{\left(\frac{s}{2}\right)^{\frac{1}{2}} y_{i}\right\} \sin \left\{\left(\frac{s}{2}\right)^{\frac{1}{2}} y_{j}\right\} \\
& \times \sin \left\{\left(\frac{s}{2}\right)^{\frac{1}{2}} y_{k}\right\} \cos \left\{\left(\frac{s}{2}\right)^{\frac{1}{2}} y_{l}\right\} \\
& G_{4}=4 \sin \left\{\left(\frac{s}{2}\right)^{\frac{1}{2}} y_{1}\right\} \sin \left\{\left(\frac{s}{2}\right)^{\frac{1}{2}} y_{2}\right\} \\
& \times \sin \left\{\left(\frac{s}{2}\right)^{\frac{1}{2}} y_{3}\right\} \sin \left\{\left(\frac{s}{2}\right)^{\frac{1}{2}} y_{4}\right\}
\end{aligned}
$$


The separatrix

$$
D=0
$$

is given by the Vandermonde determinant

$$
D=-s^{6} V\left(y_{1}, y_{2}, y_{3}, y_{4}\right)^{2}
$$

or by

$$
\begin{aligned}
D= & -4 \operatorname{det} \Gamma^{(0)} \\
D= & 27 \eta_{3}^{4}-256 \eta_{4}^{3}+128 \xi^{2} \eta_{4}^{2} \\
& -16 \xi^{4} \eta_{4}+4 \xi^{3} \eta_{3}^{2}-144 \xi \eta_{3}^{2} \eta_{4}
\end{aligned}
$$

Moreover we obtain

$$
\operatorname{det} H=\prod_{1 \leq i<j \leq 4} \frac{\sin \alpha_{i j}}{\alpha_{i j}}
$$

with

$$
\alpha_{i j}=\left(\frac{s}{2}\right)^{\frac{1}{2}}\left(y_{i}-y_{j}\right)
$$

and

$$
\operatorname{det} \Gamma\left(\xi, \eta_{3}, \eta_{4}\right)=-\frac{1}{4} D\left(\xi^{\prime}, \eta_{3}^{\prime}, \eta_{4}^{\prime}\right) \operatorname{det} H\left(\xi^{\prime}, \eta_{3}^{\prime}, \eta_{4}^{\prime}\right)^{2}
$$

In the case $N=4$ the discussion of the Schrödinger operator proceeds along the lines of section 5. The linear differential operator part in (6.1) is now (for $s=w_{3}=w_{4}=1$ )

$$
\begin{aligned}
& r_{2}=\frac{1}{2}\left(a_{7}+5\right) \tau_{2}-\frac{1}{4}\left(a_{7}^{2}-4 a_{7}+3\right) \tau_{4}+6 \\
& r_{3}=3 \tau_{3} \\
& r_{4}=\tau_{2}-\frac{1}{2}\left(a_{7}-9\right) \tau_{4}
\end{aligned}
$$

The integrability condition (6.12) is fulfilled and the solution of the differential equation (6.13) is (6.14) as for $N=3$. The potential formula (6.18) remains correct and yields the $N=4$ Sutherland model potential.

\section{Concluding remarks}

In carrying over these results to general $N$ we must clarify the meaning of the parameter $a_{7}$ in (7.1)-(7.7). Assume

$$
\begin{aligned}
\xi & =F\left(\xi^{\prime}, \eta_{3}^{\prime}, \ldots, \eta_{N}^{\prime}\right) \\
\eta_{k} & =G_{k}\left(\xi^{\prime}, \eta_{3}^{\prime}, \ldots, \eta_{N}^{\prime}\right) \\
k & \in\{3,4, \ldots, N\}
\end{aligned}
$$


to be a basic diffeomorphism satisfying the equation analogous to (4.8). Then another basic diffeomorphism is obtained from (8.1) by application of a linear map with triangular matrix

$$
\begin{gathered}
\xi=F+\sum_{\substack{\mathrm{m}=4 \\
\mathrm{~m} \text { even }}}^{\mathrm{N}} a_{2 m} G_{m} \\
\eta_{k}=G_{k}+\sum_{\substack{\mathrm{m}=\mathrm{k}+2 \\
\mathrm{~m}-\mathrm{k} \text { even }}}^{\mathrm{N}} a_{k m} G_{m}
\end{gathered}
$$

So the flat Riemannian obtained by our algorithm depends on $\frac{1}{4}(N-2)^{2}$ $\left(N\right.$ even) respectively $\frac{1}{4}(N-1)(N-3)(N$ odd $)$ parameters, which drop out of det $H$ and thereby drop out of the Schrödinger operator. Despite of the trivial appearance of these parameters in the diffeomorphism (8.2), they are connected with an annoying complication of the Riemannian as (7.7) shows.

We have not discovered yet new exactly solvable models nor have we been able to transform new known integrable models into exactly solvable forms. However, the exactly solvable form of the Sutherland models found here differs from the known one [3]. Moreover, we have shown that the formalism of the flat quadratic Lie algebraic forms implies an algorithm for the deformation of exactly solvable models into other exactly solvable models.

\section{References}

[1] F. Calogero, J. Math. Phys. 10 (1969) 219, 2197, ibid. 12 (1971) 419.

[2] M.A. Olshanetsky and A.M. Perelomov, Phys. Rep. $\underline{94}$ (1983) 313.

[3] W. Rühl and A. Turbiner, Modern Physics Letters A 10 (1995) 2213.

[4] A.V. Turbiner, Comm. Math. Phys. 118 (1988) 467; M.A. Shifman and A.V. Turbiner, Commun. Math. Phys. 126 (1989) 347.

[5] L. Brink, A. Turbiner and N. Wyllard: Hidden algebras of the (super) Calogero and Sutherland models, hep-th 9705219.

[6] A. Capella, M. Rosenbaum, A. Turbiner: Solvability of the $G_{2}$ integrable system, hep-th 9707005

[7] B. Sutherland, Phys. Rev. A 4 (1971) 2019 and 5 (1972) 1372. 